\title{
Identification of the plague reservoir in an endemic area of Zambia
}

\author{
Authors: \\ Bernard M. Hang'ombe ${ }^{1}$ \\ I. Nakamura² \\ D. Kaile ${ }^{3}$ \\ A.S. Mweene ${ }^{1}$ \\ K.L. Samui ${ }^{1}$ \\ B.S. Kilonzo ${ }^{4}$ \\ H. Sawa ${ }^{2}$ \\ C. Sugimoto ${ }^{2}$ \\ B. Wren ${ }^{5}$ \\ Affiliations: \\ ${ }^{1}$ School of Veterinary \\ Medicine, University of \\ Zambia, Zambia \\ ${ }^{2}$ Research Center for \\ Zoonosis Control, Hokkaido \\ University, Japan \\ ${ }^{3}$ Namwala District Medical \\ Officer, Namwala District, \\ Zambia \\ ${ }^{4}$ Sokoine University of \\ Agriculture, United Republic \\ of Tanzania \\ ${ }^{5}$ School of Hygiene and \\ Tropical Medicine, London, \\ United Kingdom \\ Correspondence to: \\ Bernard Hang'ombe \\ Email: \\ bernard.hang'ombe@sacids. \\ org \\ Postal address: \\ PO Box 32379, Lusaka, \\ Zambia \\ How to cite this abstract: \\ Hang'ombe, B.M., Nakamura, \\ I., Kaile, D., Mweene, A.S., \\ Samui, K.L., Kilonzo, B.S. et \\ al., 2012, 'Identification of \\ the plague reservoir in an \\ endemic area of Zambia', \\ Onderstepoort Journal of \\ Veterinary Research 79(2), \\ Art. \#464, 1 page. http:// \\ dx.doi.org/10.4102/ojvr \\ v79i2.464 \\ Note: \\ Proceedings of the \\ Conference of the Southern \\ African Centre for Infectious \\ Disease Surveillance 'One \\ Health' held at the National \\ Institute for Communicable \\ Diseases, Johannesburg, July \\ 2011.
}

Yersinia pestis, the bacterial agent of plague, is primarily a parasite of wild rodents that persists in permanent, discrete enzootic foci throughout the world. The disease is transmitted in humans by bites from fleas of wildlife rodent species. Therefore surveillance is the ultimate public health solution through plague detection in domestic dogs, other carnivores and wild rodents. The investigations of die-offs amongst plague-susceptible colonial rodents are also significant to determine the presence of $Y$. pestis in a susceptible population.

This study details the identification of the plague reservoir in a suspected endemic area of Zambia. The study was undertaken through rodent investigation for the presence of Y.pestis. A total of 105 rodents were sampled routinely and during a suspected plague period. On dissection 4 (3.81\%, $95 \%$ CI: 1.23-10.0) rodents sampled during an outbreak showed signs of spleen enlargement. The blood, liver, lymph nodes and spleen of each rodent were subjected to culture on $6 \%$ sheep blood agar and MaCconkey agar. Colonies obtained were identified as $Y$. pestis by colony morphologic features, biochemical profiles, mouse inoculation assay and polymerase chain reaction (PCR). The PCR primers used targeted the $Y$. pestis plasminogen activator gene, chromosomal ferric iron uptake regulation gene and the outer membrane protein $\mathrm{B}$ gene.

The isolates were also subjected to antibiotic sensitivity tests using the disk diffusion method on Mueller-Hinton agar with sensitivity being observed with ampicillin, amoxicillin, chloramphenicol, gentamycin, streptomycin, tetracycline and trimethoprim-sulfamethoxazole. The findings, identifies a natural reservoir of $Y$. pestis in Zambia providing the public health officials with a definite host for the control strategy. 